\title{
28 Research Square \\ DNMT1 inhibits hepatocellular carcinoma cells by altering the epigenetic modification of Marveld1 gene
}

\section{Song-Yan Zhang}

Harbin Medical University Cancer Hospital

Qian Luo

Harbin Medical Uninversity Cancer Hospital

Peng Wang

Harbin Medical University Cancer Hospital

Hao-Ran Zhao

Harbin Medical University Cancer Hospital

Hai-Tao Xu

Harbin Medical University Cancer Hospital

Liang Zhao

Harbin Medical University Cancer Hospital

Zhao-Wei Qu

Harbin Medical University Cancer Hospital

Jian-Min Sun

Harbin Medical University Cancer Hospital

Yu-Bao Zhang ( $\square$ aite_cherry@163.com )

Harbin Medical University Cancer Hospital

\section{Biao Zheng}

Shenzhen University General Hospital/Shenzhen University Clinical Medical Academy

\section{Research}

Keywords: Hepatocellular Carcinoma, Epigenetic Modification, DNMT1 Gene, Marveld1 Gene, Methylation, Proliferation

Posted Date: March 31st, 2020

DOI: https://doi.org/10.21203/rs.3.rs-19017/v1

License: @ (i) This work is licensed under a Creative Commons Attribution 4.0 International License. Read Full License 


\section{Abstract}

Background To study the effect of DNA methyltransferase (DNMT1) on Marveld1 in hepatocellular Carcinoma (HCC), and explore its role in the molecular mechanism of HCC occurrence and development.

Methods The expression levels of Dnmt1 and Marveld1 genes were detected by RT-PCR in the intraoperative pathological specimens. The human hepatoma Bel7402 and SMMC7721 cell lines down-regulated by DNMT1 gene expression were constructed by SiRNA transfection. The methylation level of Marveld1 promoter region was detected after RT-PCR and West-blot verification. The expression of Marveld1 gene, cell proliferation, invasion and change of cell cycle were detected by RT-PCR and West-blot. The expressions of P53, CyclinD1, and P21 and P16 protein were detected by West-blot method.

Results The expression of DNMT1 gene in human hepatocarcinoma tissues was higher than that in adjacent tissues. The methylation level of the Marveld1 gene promoter region in human hepatoma cell line downregulated by DNMT1 gene expression was decreased, the expression level of Marveld1 gene was increased, and the proliferation and invasion ability of cells were weakened. The cell cycle was showed as G1-S phase arrest; P53 and P16 protein expressions were up-regulated, and CyclinD1 and P21 protein expressions were down-regulated.

Conclusions Dnmt1 gene is highly expressed in human hepatocarcinoma tissues. Down-regulation of DNMT1 gene expression can decrease the methylation level of Marveld1 promoter region, up-regulate Marveld1 gene and human P53 and P16 proteins expressions, and down-regulate CyclinD1 and P21 proteins expressions, inhibit the proliferation and invasion of human hepatoma cells and can cause cell cycle G1-S arrest.

\section{Background}

Hepatocellular carcinoma (HCC) has become one of the most common malignant tumors worldwide. About 780,000 new cases of liver cancer occur each year, which accounting for $7.4 \%$ of all malignant tumors, and the mortality rate is about $9 \%$. Statistics from IARC (International Agency for Research on Cancer) showed that HCC has become the fourth leading cause of cancer death in the world ${ }^{[1]}$. HCC has a high degree of malignancy, which is easy for recurrence after surgery, and has poor prognosis. Therefore, exploring the causes of HCC and the molecular mechanism of its pathogenesis is of great value for its early diagnosis, treatment and prognosis. Existing studies have found that the occurrence of HCC is closely related to viral hepatitis, alcoholism, diet and cirrhosis, but the molecular mechanism of the disease still involves two aspects of genetic mechanism and epigenetic modification mechanism ${ }^{[2]}$. More and more evidence also indicates that epigenetic modification plays a very important role in the occurrence and development of HCC. In the study of epigenetic modification, DNA methylation research is one of the important fields of current research ${ }^{[3]}$. Studies have shown that hypermethylation inactivation of multiple tumor suppressor genes (Ecadherin, RASSF1A, GSTP, SOCS1, SFRP1, PTEN) in HCC is one of the important molecular mechanisms for the occurrence and development of $\mathrm{HCC}^{[4-6]}$.

DNA methyltransferase (DNMT) plays a key role in DNA methylation. The methylation of genomic DNA mainly occurs on the cytosine of $\mathrm{CpG}$ Island, and methyl group is added to $5^{\prime}$ end of the pyrimidine ring by the action of DNMT. The DNMTs family has approximately five members: DNMT1, DNMT2, DNMT3a, DNMT3b, and 
DNMT3L, of which DNMT1 is the most important DNA methylase and plays an important role in the maintenance of methylation status ${ }^{[7-8]}$. DNMT inhibitors have been used in a few fields such as myeloid leukemia, so the new treatment with DNMT as the entry point is a valuable direction in the field of HCC treatment.

Marveld1 (Marvel domain containing-1, MD1) gene is located in the long arm 2 region 4 band 2 sub-band of human chromosome 10 (10q24.2). The mRNA is $3238 \mathrm{bp}$ in length and has two exons. The coding region is located in the first exons, and the encoded protein product consists of 173 amino acid residues with a molecular weight of approximately $18.9 \mathrm{kDa}$. Li et al ${ }^{[9]}$ first reported the biological function of Marveld1 gene, and confirmed that it plays an important role in the regulation of malignant biological behavior of tumors in malignant tumors such as liver cancer ${ }^{[10]}$ and lung cancer ${ }^{[11]}$.

In order to study the effect of DNMT1 on Marveld1 in HCC, and explore its role in the molecular mechanism of HCC occurrence and development, and then explore a new possible method for the diagnosis and treatment of HCC, we use the method of SiRNA transfection to down-regulate the expression of DNMT1 gene in human HCC cell lines, and its effect on HCC cells was studied.

\section{Methods}

\section{Search the TCGA database}

The expression difference of DNMT1 and Marveld1 genes in liver cancer and adjacent tissues and the relationship between the expression difference and survival, the difference of methylation level of Marveld promoter region, and the related genes of DNMT1 and Marveld1 genes were examined respectively.

\section{Cell culture}

Human HCC Bel7402 and SMMC7721 cell lines were used, and DMEM medium (Gibco, Thermo Fisher Scientific Suzhou Instrument Co., Ltd.) supplemented with 10\% serum (Gibco, Grand Island, NY, USA) was used at $37^{\circ} \mathrm{C}-5 \% \mathrm{CO}_{2}$ incubator for cell culture.

\section{Construction of transfected cell lines with down-regulated expression of Dnmt1 gene}

SiRNA (Suzhou Gemma Gene) sequence:

Control group (Dnmt1-NC)

Positive-sense strand (5'-3'): UUCUCCGAACGUGUCACGUTT

Antisense strand (5'-3'): ACGUGACACGUUCGGAGAATT

Dnmt1 gene down-regulation group (Dnmt1-2505) 
Positive-sense strand (5'-3'): GGGACUGUGUCUCUGUUAUTT

Antisense strand (5'-3'): AUAACAGAGACACAGUCCCTT.

Human HCC Bel7402 and SMMC7721 cells were evenly plated into 6-well plates and cultured for 24 hours. The cell density was about $50 \%$. Premixed transfection solution was added to each well (containing 75 pmol of SiRNA, 7.5 uL of lipofectamin2000, and 200 uL of Opti-MEM medium). Further experiments were carried out after incubation for 48 hours at $37^{\circ} \mathrm{C}-5 \% \mathrm{CO} 2$.

\section{RT-PCR}

From January 2017 to December 2017, 22 pairs of HCC and adjacent cancer tissue samples were collected from the Affiliated Tumor Hospital of Harbin Medical University. All samples were preserved in liquid nitrogen immediately after collection. All the samples were confirmed to be HCC by postoperative pathology. The written informed consent of the patients for the study was obtained before surgery. The intraoperative samples used in this study were approved by the medical ethics of the Human Experimental Committee of Affiliated Cancer Hospital of Harbin Medical University.

$1 \mu \mathrm{g}$ of total RNA was extracted from tissue samples and adherent cultured cell samples with trizol, and then reverse-transcribed into cDNA by reverse transcription kit, and detected by RT-PCR using a ViiATM7 real-time PCR instrument (Applied Biosystems).

Dnmt1 gene primer sequence:

Upstream 5'-AGGCGGCTCAAAGATTTGGAA-3'

Downstream 5'-GCAGAAATTCGTGCAAGAGATTC-3'

Marveld1 gene primer sequence:

Upstream 5'- ACTGAGAAGTCCCGCTGTTACAG -3'

Downstream 5'- GGGATGCTGGGAATCTTAAGG-3'

GAPDH Primer Sequence:

Upstream 5'-AGGCTGTGGGCAAGGTCATC-3'

Downstream 5'-TCAGGTCCACCACTGACACG-3'

The human hepatocellular carcinoma cell line with down-regulated expression of DNMT3a and DNMT3b was constructed using the SiRNA transfection method with the same expression of down-regulation of Dnmt1, and the sequence of SiRNA (Suzhou Gemma gene):

Dnmt-NC (control group)

Positive-sense strand (5'-3'): UUCUCCGAACGUGUCACGUTT 
Antisense strand (5'-3'): ACGUGACACGUUCGGAGAATT

DNMT3a-SI

Positive-sense strand (5'-3'): CGGCUCUUCUUUGAGUUCUTT

Antisense strand (5'-3'): AGAACUCAAAGAAGAGCCGTT

DNMT3b-SI

Positive-sense strand (5'-3'): GUGAGGAACAUCACAAAGATT

Antisense strand (5'-3'): UCUUUGUGAUGUUCCUCACTT

RT-PCR was used to detect the down-regulation of DNMT3a and DNMT3b expression and the corresponding Marveld1 gene expression change.

DNMT3a Primer Sequence:

Upstream: 5'-CCGATGCTGGGGACAAGAAT -3'

Downstream: 5'-CCCGTCATCCACCAAGACAC -3'

DNMT3b primer sequence:

Upstream: 5'- AGGGAAGACTCGATCCTCGTC- 3'

Downstream: 5'- GTGTGTAGCTTAGCAGACTGG-3'

Marveld1 Primer Sequence:

Upstream: 5' -ACTGAGAAGTCCCGCTGTTACAG-3'

Downstream: 5'- GGGATGCTGGGAATCTTAAGG-3'

GAPDH Primer Sequence:

Upstream: 5'- AGGCTGTGGGCAAGGTCATC- 3'

Downstream: 5' -TCAGGTCCACCACTGACACG- 3'

\section{Marveld1 gene promoter region methylation level sequencing detection}

Marveld1 gene promoter region methylation level sequencing detection

The 1ug genomic DNA was extracted, and the bisulfite conversion and purification and collection were carried out according to the kit instructions (QIAGEN, No. 59824). The sequence of the Marveld1 gene promoter region 
was found in $\mathrm{NCBI}$ and designed the primers:

Upstream 5'-GCCAGGGCAAGCAGGAGGTGG-3'

Downstream 5'-CTGCTGAGACTAAGACGTGG-3'

After PCR amplification, the amplification products were sequenced to determine the methylation level of the Marveld1 gene promoter region, and the sequence and $\mathrm{CpG}$ island sites were as follows:

$1 \mathrm{Cpg} \quad 2 \mathrm{Cpg} \quad 3 \mathrm{CpG} \quad 4 \mathrm{CpG} \quad 5 \mathrm{CpG}$

CGAGTGGAACGAGTGGCGGTGCCTCTGGTGGGTGATGGACTCTTACTCCGACATGGGTAACTCGGACAG

$6 \mathrm{Cpg} 7 \mathrm{Cpg} \quad$ 8CpG

CTTTGGACAGGACGAGGCGGGCCCTGGCAGGAGACTGCGGATGTCCAGGTGACAGTGGATGCCAGTTCA

9CpG10CpG $\quad 11 \mathrm{Cpg} \quad 12 \mathrm{CpG}$

AAACCAGCACTGCTCTCCTCCCG CGCCCAGGAAGAGCCCAGAGGCCCGGGGTGGGCGCTATGGCAGG

$13 \mathrm{CpG} \quad 14 \mathrm{CpG}$

AAAGAGGGAAAAGGAGGAGGCAGGGCGGCAGGGGCG

\section{Western-blot}

Cell count: Taken the same number of cells and added with RIPA lysate (containing 5\% Doc, $1 \%$ PMSF, $1 \%$ pepstain $A, 1 \%$ ribonuclease) at a concentration of $1 \times 106$ cells $/ 100 \mu l$, and after lysed at $4^{\circ} \mathrm{C}$ for 30 minutes, centrifuged at 12,000 rpm and then taken the supernatant protein solution. SDS-acrylamide gel electrophoresis was carried out and imprinted on a 0.2 um PVDF membrane. Washed with $0.1 \%$ PBST after sealed for 1 hour with $5 \%$ skim milk, and then immersed in $0.1 \%$ PBST diluted primary antibody overnight; washed with $0.1 \%$ PBST, and then immersed in secondary antibody for 1 hour, then washed with $0.1 \%$ PBST, and added with developer for development by ECL Western Blot Analysis System (Amersham Biosciences, Chalfont St. Giles, Buckinghamshire, UK), and grayscale analysis was conducted.

\section{Measurement of cell proliferation ability by MTT assay}

The cells were plated into the 96 -well plates with 1500 cells/well, cultured and adherent with $200 \mu$ l of DMEM medium, and then added $100 \mu \mathrm{l}$ of medium containing $10 \%$ MTT to each well at $0 \mathrm{~h}, 24 \mathrm{~h}, 48 \mathrm{~h}, 72 \mathrm{~h}$ and $96 \mathrm{~h}$, respectively, after cultured for 4 hours, $100 \mu \mathrm{l}$ of DMSO was added to each well, and the OD value was read at a wavelength of $450 \mathrm{~nm}$ by a micro plate after shaking. The OhOD value was used as the baseline, and the ratio of the OD value at each time point to the OD value at Oh was the cell proliferation rate, and the proliferation curve of each group was plotted. 


\section{Changes of cell invasion ability after down-regulation of Dnmt1 gene expression was measured by Transwell method}

The upper chamber of Transwell plate (3428, costar) was evenly spread with glue, and then spread with cells with $3 \times 105$ cells/well in each well, and $1.5 \mathrm{ml}$ of DMEM medium containing $0.5 \%$ serum was added, and $2.6 \mathrm{ml}$ of medium containing $10 \%$ serum was added to the lower chamber; After cultured at $37^{\circ} \mathrm{C}-5 \%$ for 24 hours, photographs were taken under a crystal violet staining microscope. A total of 4 visual fields were selected randomly from each group for counting.

\section{Determination of Cell Cycle Changes after Down-regulation of Dnmt1 Gene Expression with flow cytometry}

The cells were cultured in an adherent manner without EDTA trypsinization, and then the cells were collected after centrifugation, and after washed with PBS, the cells were fixed with $75 \%$ ethanol at $4{ }^{\circ} \mathrm{C}$ for 24 hours; then washed with PBS, PI staining was performed for $30 \mathrm{~min}$, and the cell cycle was measured by flow cytometry after washing with PBS.

\section{Statistical analysis}

SPSS 17.0 statistical software was used for statistical analysis of each experimental result. The result data was expressed as $X \pm s$. $P<0.05$ was considered statistically significant.

\section{Results}

\section{TCGA database search results}

The search results of TCGA database showed that the expression of DNMT1 gene in HCC was higher than that in adjacent tissues, the expression of Marveld1 gene was lower than that in adjacent tissues (Figure 1A), and the methylation level of promoter region was higher than that of adjacent tissues (Figure 1B). The changes of expression of DNMT1 and Marveld1 gene were related with poor prognosis (Figure 1C). There is currently no data on the association between DNMT1 gene and Marveld1 gene expression.

Paired analysis of RT-PCR results of 22 clinical samples showed that the expression level of Dnmt 1 gene in HCC tissues was higher than that in adjacent tissues, and the expression of Marveld1 gene in HCC tissues was lower than that in adjacent tissues (Figure 1D).

\section{Construction of human HCC cell line with down-regulated Dnmt1 gene expression}

After transfection with Si-RNA, the RNA and protein expression of Dnmt1 gene in the experimental group was significantly decreased (Figure 2A). 


\section{Detection of Marvel1 gene expression level}

Detection of methylation level of Marveld1 gene promoter region

The $14 \mathrm{CpG}$ islands in the promoter region of Marveld1 gene were sequenced, and the results showed the Bel7402 methylation CpGs rate in NC group was $(57.1 \pm 2.38) \%$ and $(43.6 \pm 6.61) \%$ in the SI group, and the SMMC7721 methylation CpGs rate in NC group was $(40.7 \pm 2.14) \%$ and $(32.9 \pm 4.42) \%$ in the SI group; this result indicates that the number of methylation in human HCC cells with down-regulated Dnmt1 expression was lower than that in the control group, and the methylation level of the Marveld1 gene promoter region was decreased (Figure 2B and 2C).

Marvld1 gene expression detection

The RNA and protein expression of Marveld1 gene in human HCC cells with down-regulated Dnmt1 expression was up-regulated (Figure 3A, Figure 3B).

\section{Changes of proliferative capacity of human HCC after down- regulation of Dnmt1 gene expression by MTT assay}

In the experimental group, the cell proliferation ratio was higher than that of the control group in the $0 \mathrm{~h}-96 \mathrm{~h}$ time period; at $72 \mathrm{~h}$, the Bel7402 cell proliferation ratio in the NC group was $(4.9670 \pm 0.1597)$ and

(2.9676 \pm 0.3614$)$ in the SI group; SMMC7721 cell proliferation ratio in the NC group was $(3.2734 \pm 0.1394)$ and (2.5646 \pm 0.0789$)$ in the SI group. After down-regulation of Dnmt1 gene expression, the proliferative capacity of human HCC was decreased significantly (Figure $3 \mathrm{C}$ ).

\section{Detection of the change of invasive ability of HCC cells after down-regulation of Dnmt1 gene expression by Transwell assay}

The number of invading cells of Bel7402 in the NC group was $(155.25 \pm 15.97)$ and $(65.00 \pm 4.55)$ in the SI group; the number of SMMC7721 invasive cells in the NC group was (102.70 \pm 12.90$)$ and $(41.00 \pm 6.27)$ in the SI group. After down-regulation of Dnmt1 gene expression, the invasive ability of HCC cells was weakened (Figure 3D and $3 \mathrm{E})$.

\section{Detection of change of cell cycle after down-regulation of Dnmt1 gene expression by flow cytometry}

After down-regulation of Dnmt1 gene expression, $\mathrm{HCC}$ cells were increase in G1 phase, and decreased in S phase, suggesting that down-regulation of Dnmt1 gene expression may cause cell cycle G1-S arrest in HCC cells (Figure 3F). 


\section{West-Blot detection of P53, CyclinD1, P21 and P16 protein expression}

After transfection with Si-RNA, the expression of P53, P21 and P16 proteins was up-regulated, and the expression of CyclinD1 protein was down-regulated (Figure 4).

After transfection with Si-RNA, the RNA expression of DNMT3a and DNMT3b genes in the experimental group was significantly decreased $\left({ }^{*} p<0.05\right)$, Figure 5.

However, the Marveld1 gene of human hepatocytes with down-regulated DNMT3a and DNMT3b gene expression was not significantly changed compared with the Marveld1 gene of human hepatocytes with downregulated DNMT1 gene expression. Therefore, human hepatocellular carcinoma cells with down-regulated DNMT1 gene expression were selected as experimental subjects (\#p>0.05), Figure 6.

\section{Discussion}

DNMT, as the key factor in the regulation of DNA methylation in tumor tissues, can affect the biological behavior of tumor cells by regulating the degree of methylation of gene promoters with different functional ${ }^{[12]}$. The DNMTs family has approximately five members: DNMT1, DNMT2, DNMT3a, DNMT3b, and DNMT3L, of which DNMT1 is the most important DNA methyltransferase and plays an important role in methylation status maintenance; deletion of DNMT1 may lead to decreasing of DNA methylation, affect the transcription of genes, and then regulate cell growth and differentiation.

Proteins containing the MARVEL domain are evolutionarily conserved and widely expressed in normal adult tissues. They involve a variety of biological processes, including cell cycle progression, chemotactic activity, tight junctions, and clathrin-mediated endocytosis ${ }^{[9,13-16]}$. As a member of this family, the research on the molecular function of Marbeld-1 is limited so far. Studies have shown that the expression of Marveld-1 gene in liver cancer and lung cancer tissues is lower than that in adjacent tissues. Overexpression of Marveld- 1 gene can inhibit the proliferation of tumor cells, up-regulate P53 and P16 genes, and can down-regulate the expression of CyclinD1 gene, Marveld-1 can affect the G1-S phase checkpoint-associated proteins of the cell cycle mentioned above, and then lead to G1-S phase arrest of Marveld-1 overexpressing cells, thus exert the function of tumor suppressor gene ${ }^{[10-11]}$.

The data in the TCGA database showed that the DNMT1 gene was highly expressed in HCC tissues and low in Marveld1 gene, which was consistent with the clinical sample paired PCR results in this study. Marveld1 gene was hypermethylated in HCC tissues, and the survival of the samples with low expression of Dnmt1 gene was longer than that of the samples with high expression of Dnmt1 gene, suggesting that down-regulation of DNMT1 gene expression can up-regulate the expression of Marveld1 gene, and the results of inhibiting the malignant biological manifestations of HCC may have potential clinical application value. There is no data on the relationship between Marveld1 gene and DNMT1 gene in TCGA database. This study showed that the down-regulation of Dnmt1 gene can up-regulate the expression of Marveld1 gene by down-regulating the methylation level of Marveld1 gene promoter region, and there is certain correlation between the expressions of two genes. 
This study found that in human HCC tissues, the expression of Dnmt1 gene in HCC tissues was higher than that in adjacent tissues. Down-regulation of the expression of Dnmt1 gene in HCC cells could down-regulate the methylation level of the promoter region of Marveld1 gene. The expression of Marveld1 gene can be upregulated by the epigenetic modification, and can inhibit the proliferation and invasion of HCC cells that consistent with the existed study on the overexpression of Marveld1 gene ${ }^{[10]}$. Up-regulated P53, P21 and P16 genes, down-regulated the expression of CyclinD1 gene, and affecting the G1-S phase checkpoint-associated proteins in the cell cycle, thus leading to G1-S phase arrest in cells. The Dnmt1 gene can change pathway, cause the epigenetic modification of the Marveld1 gene through a promoter methylation level, thus resulting in up-regulation of its expression and enhancing the function of the tumor suppressor gene.

\section{Conclusions}

DNMT inhibitors have been used in clinical applications, and the main therapeutic areas are bladder cancer and myeloid leukemia. The results of this study suggest that DNMT inhibitors may have some application prospects in the field of HCC at the in vitro cytological level, and related cells, animals and clinical trials may be a valuable research direction.

\section{Abbreviations}

DNMT: DNA methyltransferase; HCC: hepatocellular carcinoma; MD1: Marvel domain containing-1.

\section{Declarations}

\section{Ethics approval and consent to participate}

Not applicable.

\section{Consent for publication}

Not applicable.

\section{Availability of data and materials}

All data generated or analysed during this study are included in this published article.

\section{Competing interests}

The authors declare that they have no competing interests.

\section{Funding}

This study is funded by Harbin Medical University Cancer Hospital HaiYan Science Foundation Key Program, No. JJZD2014-06.

\section{Authors' contributions}


SYZ and QL: conceived and designed the experiments, performed the experiments.

YBZ and BZ: performed the experiments, analyzed the data, and wrote the manuscript.

PW, HRZ, HTX, LZ, ZWQ and JMS: analyzed the data and proofed the manuscript.

All authors have proofed the article.

\section{Acknowledgements}

Not applicable.

\section{References}

[1] Jemal A, Bray F, Center MM. Global cancer statistics. A Cancer J Clinicians 2011; 61(2):69-90.

[2] Eden A, Gaudet F, Waghmare A, Jaenisch R. Chromosomal instability and tumors promoted by DNA hypomethylation. Science 2003; 300(5618):455.

[3] Li XD, Wang YW, Hu J. Progress on epigenetic research of liver cancer. Journal of Modern Oncology 2016; 24(09):1501-1504.

[4] Calvisi DF, Ladu S, Gorden A, Farina M, Lee JS, Conner EA, Schroeder I, Factor VM, Thorgeirsson SS. Mechanistic and prognostic significance of aberrant methylation in the molecular pathogenesis of human hepatocellular carcinoma. J Clin Invest 2007; 117(9):2713-22.

[5] Tischoff I, Tannapfe A. DNA methylation in hepatocellular carcinoma. World J Gastroentero/ 2008; 14(11):1741-8.

[6] Lou C, Yang B, Gao YT, Wang YJ, Nie FH, Yuan Q, Zhang CL, Du Z. Aberrant methylation of multiple genes and its clinical implication in hepatocellular carcinoma. Chinese Journal of Oncology 2008; 30(11):831-6.

[7] Bird A. DNA methylation patterns and epigenetic memory. Genes Dev 2002; 16(1):6-21.

[8] Liang G, Chan MF, Tomigahara Y, Tsai YC, Gonzales FA, Li E, Laird PW, Jones PA. Cooperativity between DNA methyltransferases in the maintenance methylation of repetitive elements. Mol Cell Biol 2002; 22(2):48091.

[9] S Wang ,Y Li ,F Han, et al. Identification and characterization of MARVELD1, a novel nuclear protein that is down-regulated in multiple cancers and silenced by DNA methylation. Cancer Letters 2009; 282 (1):77-86

[10] Yu L, Zhang Y, Hu J, et al. MARVELD1 inhibited cell proliferation and enhance chemosensitivity via increasing expression of p53 and p16 in hepatocellular carcinoma. Cancer Sci 2012; 103(4):716-22.

[11] M Shi, S Wang, Y Yao, et al. Biological and clinical significance of epigenetic silencing of MARVELD1 gene in lung cancer. Scientific Reports 2013; 4:7545-7545. 
[12] Schutte M, Hruban RH, Geradts J, Maynard R, Hilgers W, Rabindran SK, Moskaluk CA, Hahn SA, SchwarteWaldhoff I, Schmiegel W, Baylin SB, Kern SE, Herman JG. Abrogation of the Rb/p16 tumor-suppressive pathway in virtually all pancreatic carcinomas. Cancer Res 1997; 57(15):3126-30.

[13] Mimori K, Shiraishi T, Mashino K et al. MAL gene expression in esophageal cancer suppresses motility, invasion and tumorigenicity and enhancesapoptosis through the Fas pathway. Oncogene 2003; 22: 3463-71.

[14] Shi Y, Ding X, He ZH et al. Critical role of TRPC6 channels in G2 phasetransition and the development of human esophageal cancer. Gut 2009; 58:1443-50.

[15] Han W, Lou Y, Tang V et al; Molecular cloning and characterization of chemokine-like factor 1 (CKLF1), a novel human cytokine with uniquestructure and potential chemotactic activity. Biochem J 2001; 357: 1273520 .

[16] Martı́n-Belmonte F, Martı́nez-Mena'rguez JA, Aranda JF et al. MAL regulates clathrin-mediated endocytosis at the apical surface of Madin-Darby canine kidney cells. J Cell Bio/2003; 163: 155-64.

\section{Figures}

A

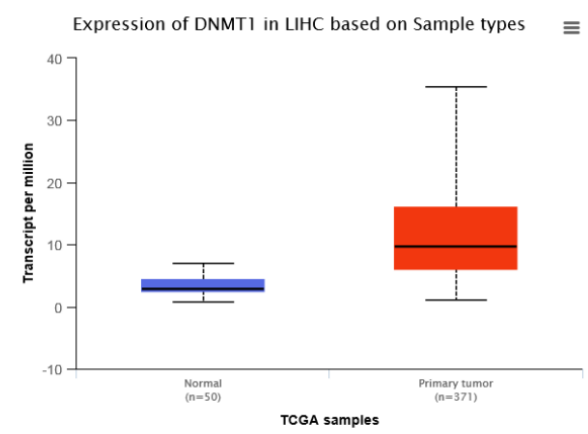

$p=5.71189999964972 E-07$

C

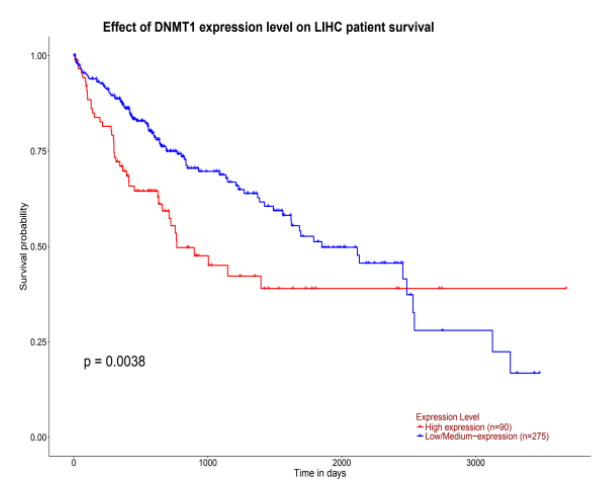

$\mathrm{B}$

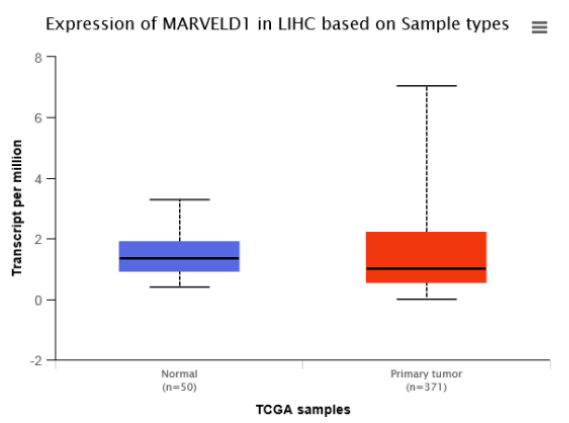

$p=1.62447832963153 E-12$

$\mathrm{D}$

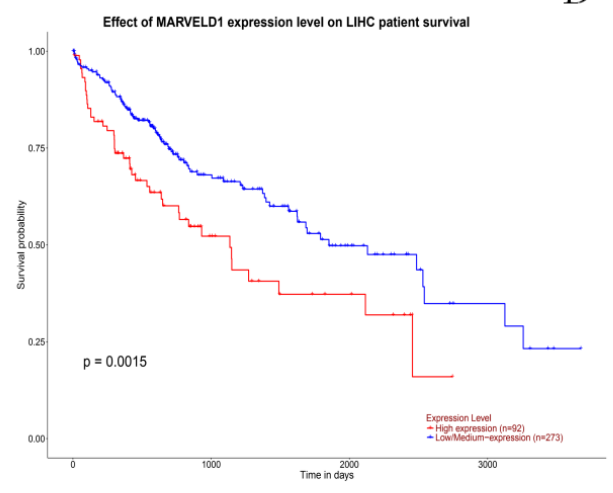

$\mathrm{p}=2.75649947312218 \mathrm{E}-10$
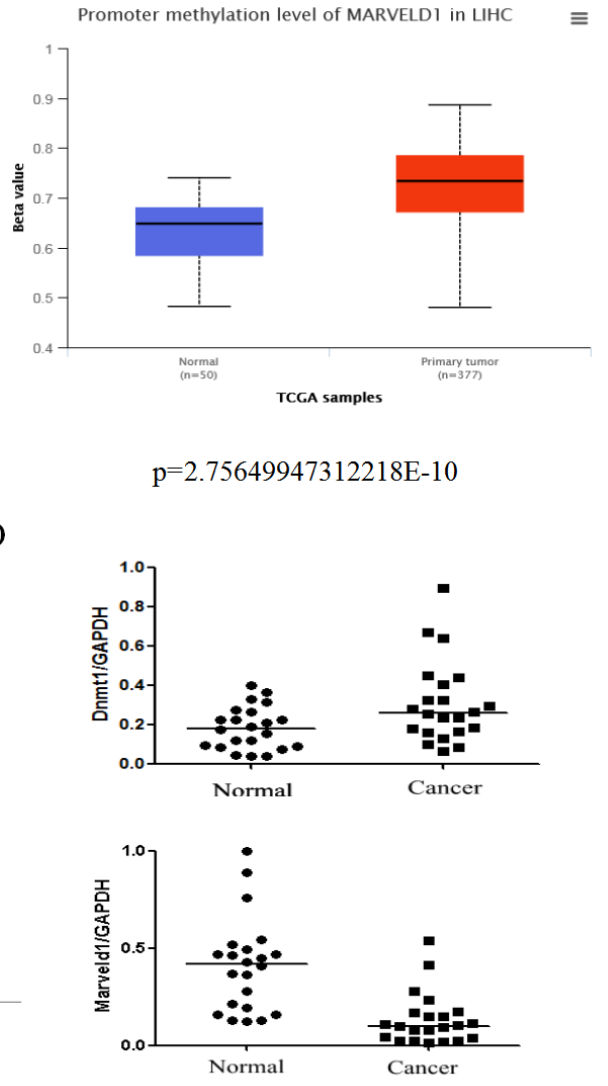

\section{Figure 1}

TCGA database search results and Paired RT-PCR detection results 
A

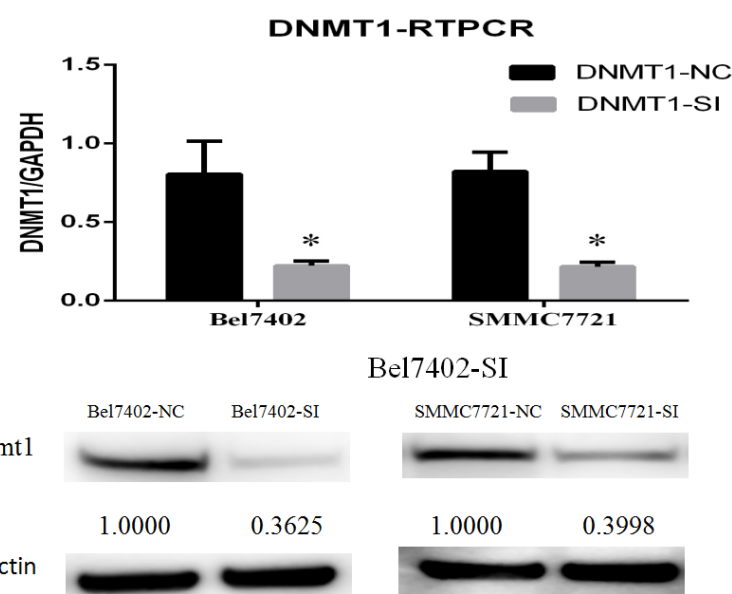

D

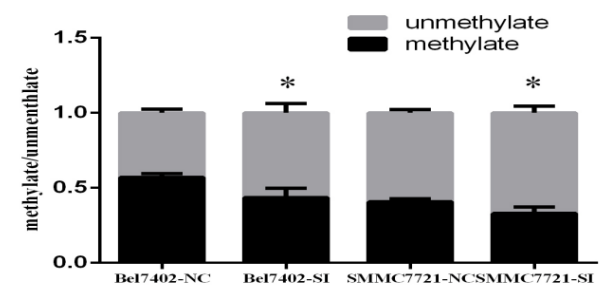

C

Methylation

CDG Position
Unmethylated Unmethylated
Methylated
Not present

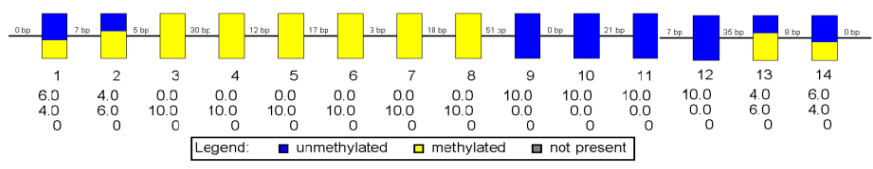

Bel7402-NC

Methylation

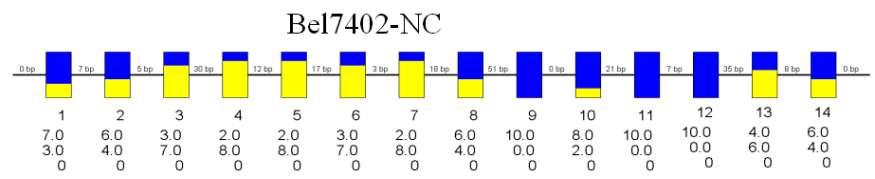

CpG Position

Unmethylated
Methylated

Not present

Methylation

CpG Position

Unmethylated
Methylated

Methylated
Not present

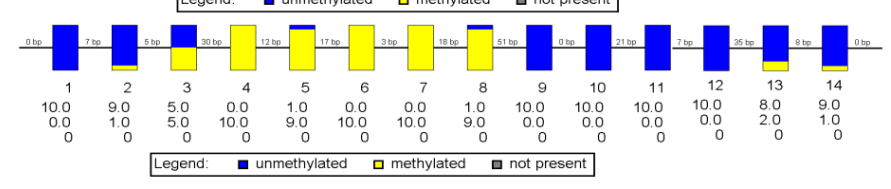

SMMC7721-NC

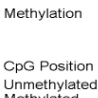
Unmethylate Methylated
Not present

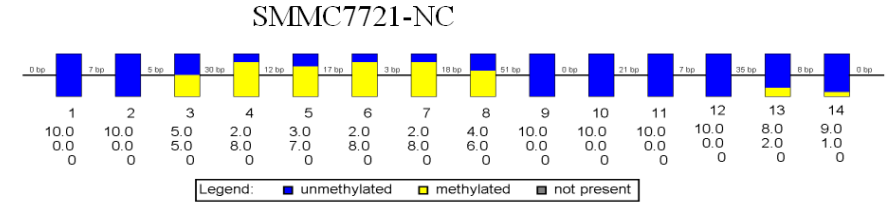

SMMC7721-SI

\section{Figure 2}

A: After transfection, the RNA and protein expression of Dnmt1 gene in the experimental group was decreased.

$B$ : The number of methylation in human hepatocellular carcinoma cells with down-regulated Dnmt1 expression was lower than that in the control group; ${ }^{\star} \mathrm{P}<0.05$; $\mathrm{C}$ : Marveld1 gene promoter region methylation sequencing; D: Methylation level of the Marveld1 gene promoter region 
A

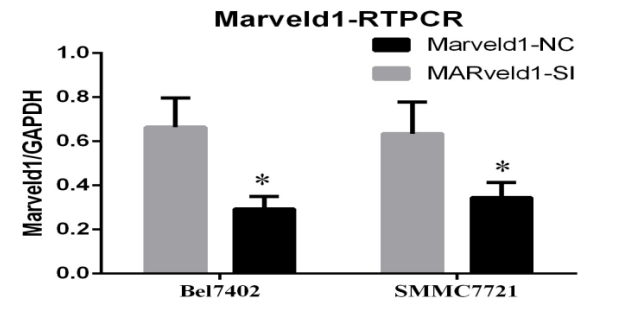

C
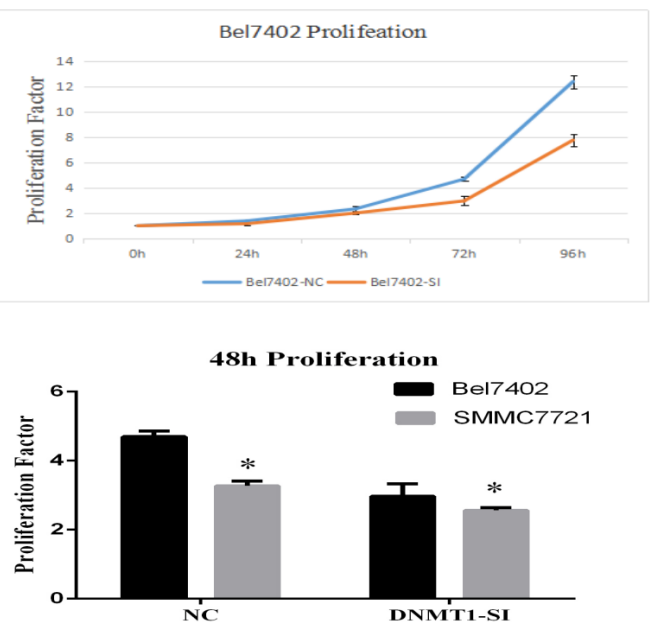

E Bel7402-NC

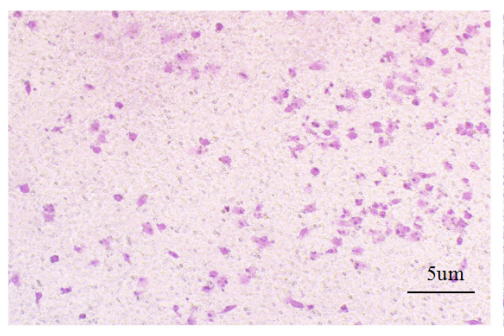
SMMC7721-NC

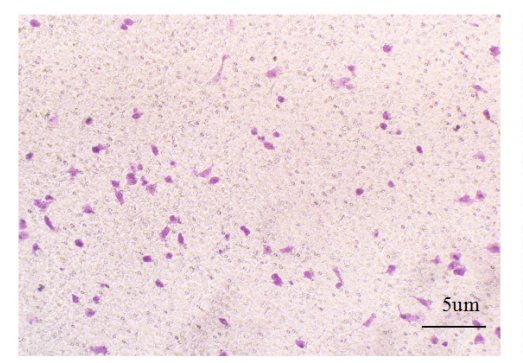

B
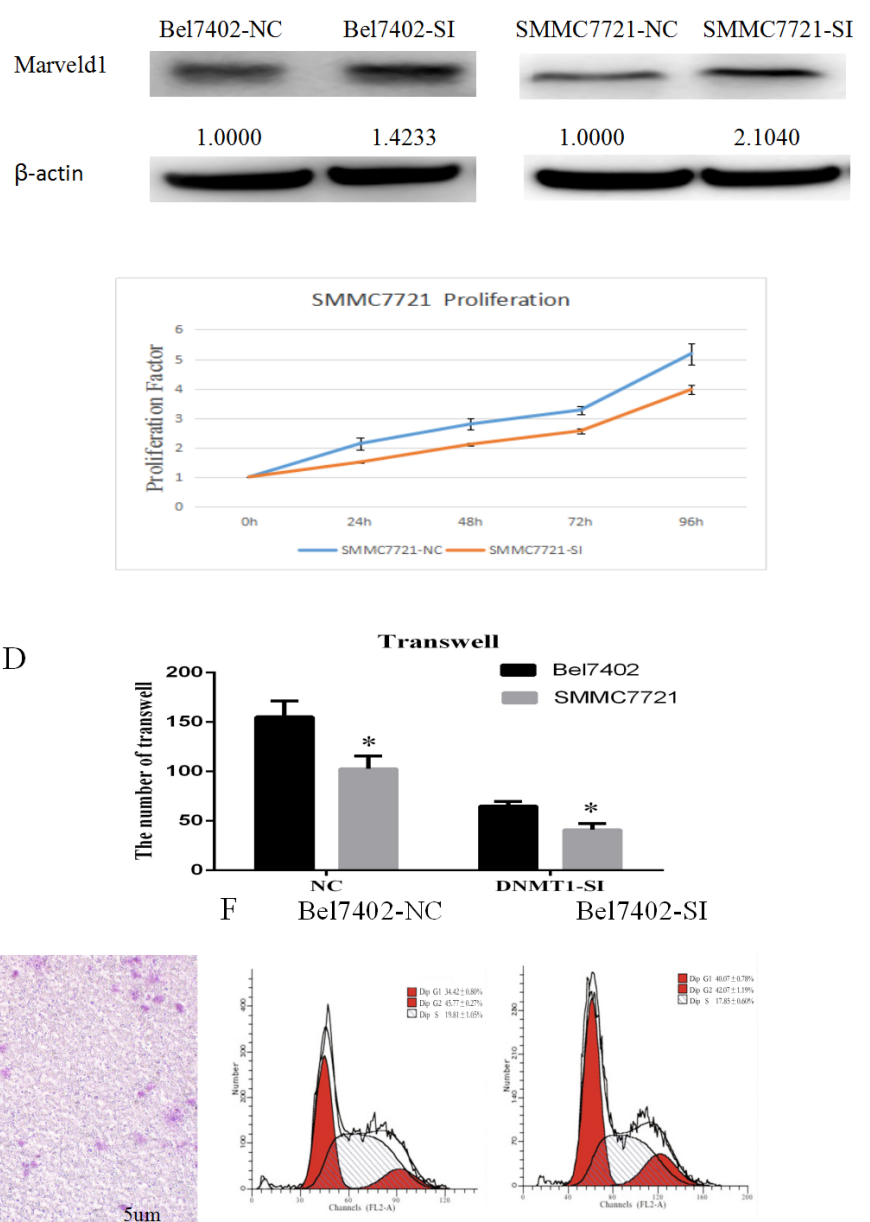

Sum

SMMC7721-NC

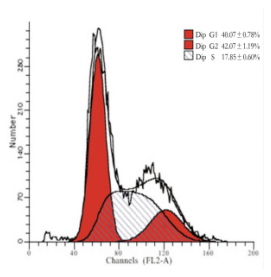

SMMC7721-SI
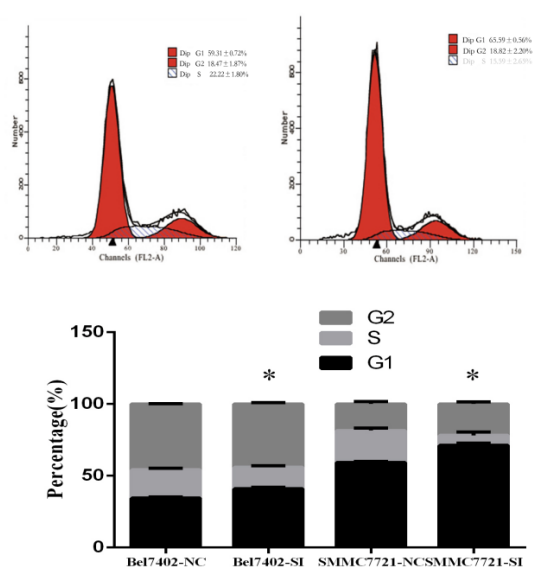

\section{Figure 3}

A: RT-PCR detection of Marveld1 gene expression; B: Western-Blot detection of Marveld1 gene expression; C: Cell proliferation assay; D/E: Cell invasion assay; F: Cell cycle detection. 
Bel7402-NC Bel7402-SI

P53

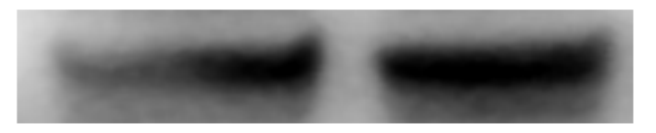

1.0000

1.4258

CyclinD1

P21

1.0000

0.6298

1.0000

1.7518

P16

1.0000

2.7922

$\beta$-actin

P21

P16

$\beta$-actin
P53

SMMC7721-NC SMMC7721-SI

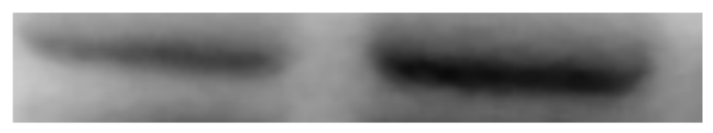

1.0000

1.5561

CyclinD1
1.0000
0.6346

1.0000

1.5993

1.0000

1.5698

Figure 4

Western-Blot detection of p53 pathway-related genes

DNMT3a-RTPCR

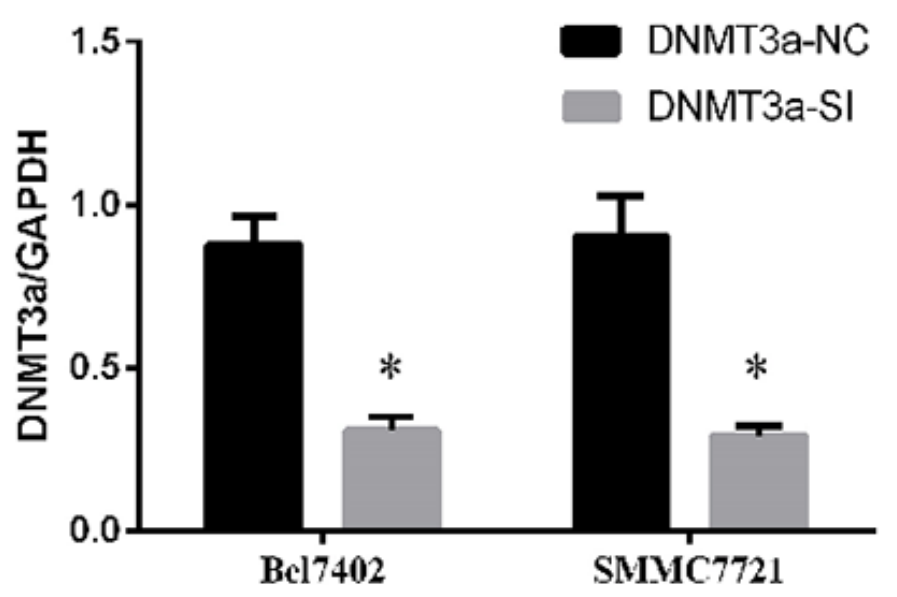

DNMT3b-RTPCR

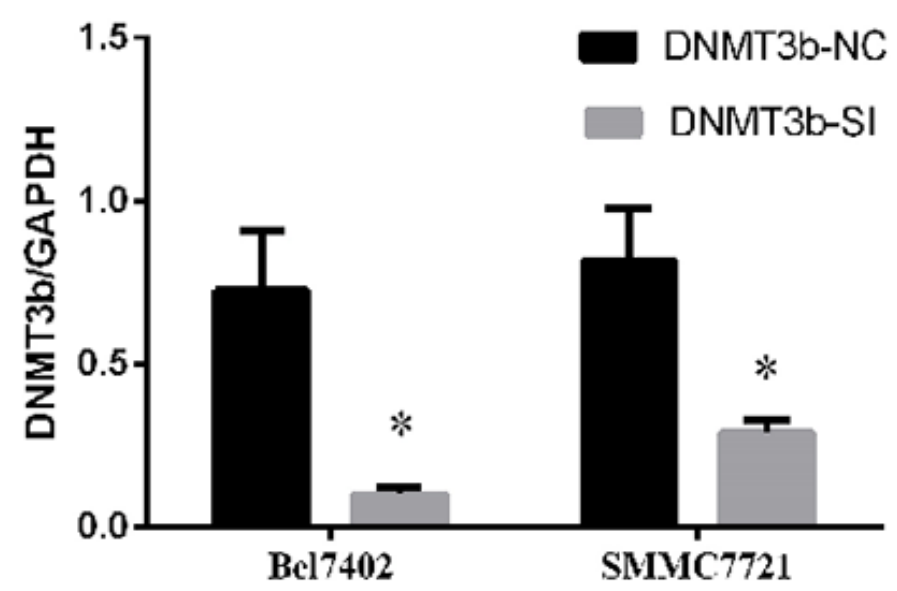


Figure 5

The expressions of DNMT3a and DNMT3b after SiRNA transfection

DNMT3a-Marveld1 RTPCR

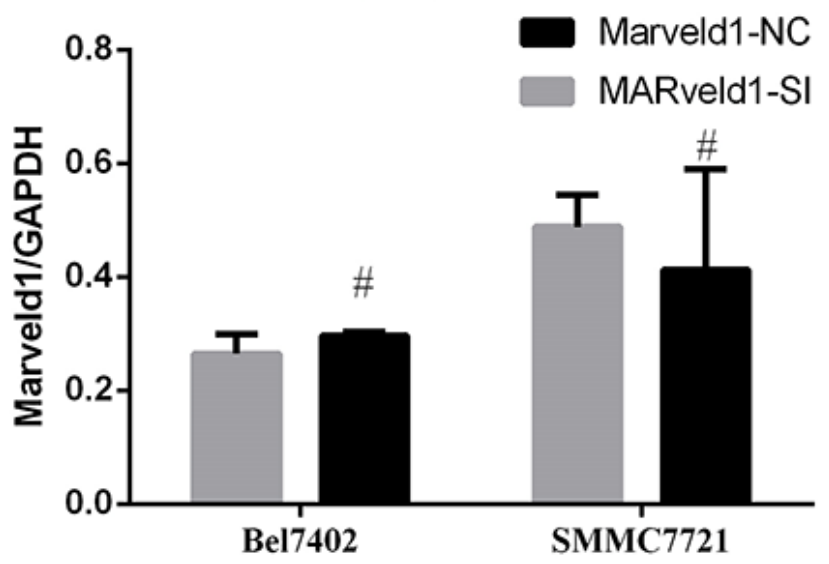

DNMT3b/Marveld1 RTPCR

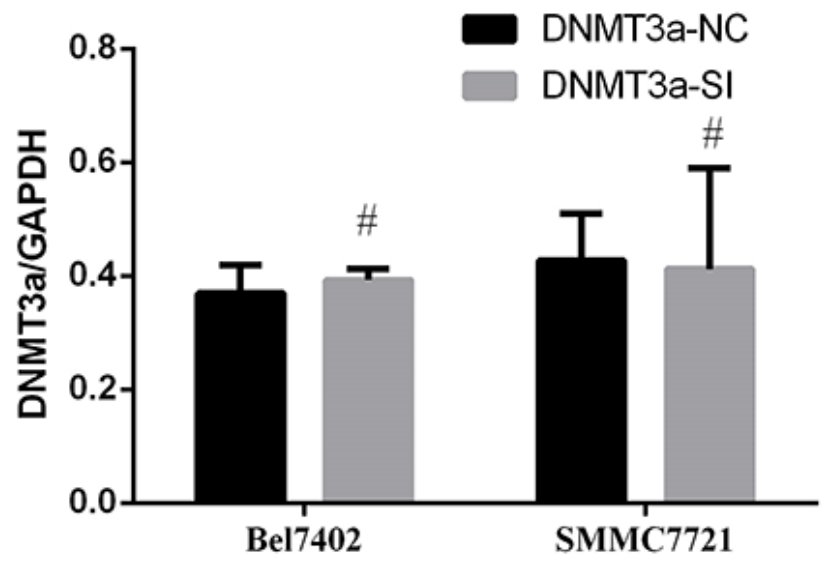

Figure 6

DNMT3a and DNMT3b RTPCR. 